\title{
SiSTEMAS ELECTORALES Y SU INFLUENCIA EN EL DISEÑO DE LA POLÍTICA AMBIENTAL
}

\section{Raúl Alberto Ponce Rodríguez y Alan Adrián Rodríguez Hernández}

Fecha de recepción: 30 de octubre de 2019. Fecha de aceptación: 4 de mayo de 2020.

$$
\text { http://doi.org/10.22201/iiec.20078951e.2020.203.69499 }
$$

\begin{abstract}
Resumen. Se presenta el desarrollo de un análisis comparativo del impacto de los sistemas electorales mayoritarios y de representación proporcional en una política de impuestos que busca reducir la contaminación. Los resultados obtenidos son los siguientes: en un sistema electoral mayoritario, la regulación ambiental es extrema con impuestos muy bajos o altos en comparación a la política socialmente óptima; en un sistema de representación proporcional, la política ambiental es moderada (el impuesto se encuentra en una posición intermedia con relación en los impuestos implementados en un sistema mayoritario); y la política ambiental en sistemas de representación proporcional se aproxima más a la política socialmente óptima. Instituciones electorales explican estos resultados a través del número de partidos efectivos y la negociación legislativa en el Congreso.
\end{abstract}

Palabras clave: contaminación; impuestos; política ambiental; regulación; modelo teórico; sistema electoral; democracia.

Clasificación JEL: D72; E62; H23; Q56; Q58.

\section{Electoral Systems AND THEIR INFLUENCE on Environmental Policy Design}

\begin{abstract}
This article undertakes a comparative analysis of the respective effects of majority and proportional representation electoral systems on tax policies designed to reduce pollution. The following findings were obtained: in a majority electoral system, environmental regulation is extreme with very low or high taxes compared to a socially optimal policy; in a proportional representation system, environmental policy is moderate (taxes are moderate compared to taxes implemented in a majority system); and environmental policy in proportional representation systems more closely resembles a socially optimal policy. Electoral institutions account for these findings, which are due to the number of effective parties and legislative negotiation in Congress.
\end{abstract} Key Words: pollution; taxes; environmental policy; regulation; theoretical model; electoral system; democracy.

\footnotetext{
${ }^{a}$ Universidad Autónoma de Ciudad Juárez (UACJ), México. Correos electrónicos: rponce@uacj.mx y alan.rodriguez@uacj.mx, respectivamente.
} 


\section{INTRODUCCIÓN}

Existe una abundante literatura que muestra que la política de redistribución del gobierno, la provisión de bienes públicos, y la regulación económica son, en parte, determinadas por aspectos políticos tales como la competencia electoral, la negociación legislativa entre el Poder Ejecutivo y el Congreso, las preferencias sobre la política económica de los partidos, la participación electoral de los votantes y, en general, por la ingeniería electoral de una democracia (véase Mueller, 2003). El caso de la regulación ambiental que busca establecer reglas de operación a los agentes económicos sobre las externalidades negativas causadas por la contaminación ambiental no es la excepción.

A pesar del creciente interés en el área de la nueva economía política para analizar el esfuerzo del gobierno en regular las actividades económicas asociadas a externalidades negativas como la contaminación, se conoce muy poco sobre el papel del sistema electoral para determinar el nivel de regulación ambiental del gobierno. No obstante, el tema es importante, ya que a nivel internacional existe una importante heterogeneidad en el diseño e implementación de la política ambiental. Mientras que hay países que están dispuestos a subscribirse a protocolos internacionales que buscan regular la contaminación, existen otros que han rechazado adoptar sus recomendaciones. La evidencia conduce a la siguiente pregunta de interés: ¿qué explica que ciertos países busquen aminorar los efectos negativos de la contaminación, mientras que otros adoptan regulaciones ambientales más flexibles?

El análisis económico tradicional -que considera que el gobierno es controlado por un planeador social benevolente- sugiere que la disposición de cada país de establecer una regulación ambiental se relaciona con un análisis costo-beneficio que incorpora la distribución de los beneficios y costos marginales sociales de las actividades que contaminan. Sin embargo, este análisis tradicional no incorpora que, en la práctica, los gobiernos podrían no tener incentivos a utilizar las curvas de beneficios y costos marginales sociales para guiar el diseño de la regulación ambiental. Este podría ser el caso, si las curvas de los beneficios y costos marginales electorales que afectan el diseńo de la política económica del gobierno no coinciden con las curvas de beneficio y costo marginal de la sociedad.

Debido a que la conformación de un gobierno requiere que un partido gane una elección, es importante destacar que los partidos integrantes del gobierno tienen incentivos a adoptar aquellas políticas económicas que maximizan el número de votos que un partido puede recibir en una elección. Si bien es cierto, los incentivos electorales no son los únicos determinantes del diseño 
de la política económica de un gobierno en una democracia, también habría que reconocer que la evidencia muestra que la competencia electoral sí es un determinante importante de la política económica (véase Mueller, 2003).

En este trabajo se reconoce que los incentivos electorales para la formación de un gobierno podrían ser fundamentales para explicar el diseño de la política económica de un gobierno democráticamente electo. Por ello, se buscó estudiar cómo ciertas instituciones del sistema electoral de una democracia pueden ejercer influencia para determinar una política de regulación ambiental flexible (que tolere altos niveles de contaminación) o una regulación ambiental restrictiva (que sólo tolere niveles bajos de contaminación).

En este contexto, es importante reconocer que las instituciones del sistema electoral de una democracia están vinculadas a las políticas ambientales adoptadas en ella, ya que estas instituciones determinan el número de partidos que compiten en una elección, así como el nivel de representación de cada uno en el Congreso. Debido a que la política ambiental requiere la aprobación mayoritaria simple o calificada, entonces el tipo de sistema electoral adoptado -ya sea un sistema electoral mayoritario, de representación proporcional, o mixto-, creará incentivos (o barreras) para que el partido (o los partidos) en el gobierno adopten una política de regulación ambiental flexible o una regulación ambiental restrictiva.

En el presente trabajo se plantea la siguiente pregunta: ¿cómo afecta la adopción de un sistema electoral al diseño de la regulación ambiental?, es decir, ¿̇un sistema electoral mayoritario produce el mismo tipo de regulación ambiental que un sistema electoral de representación proporcional? En la investigación se desarrolla un análisis teórico comparado sobre el tipo de regulación ambiental que surge en una democracia con un sistema electoral mayoritario o de representación proporcional. Así, se desarrolló un modelo teórico de competencia electoral en el que el gobierno selecciona un impuesto que busca reducir la contaminación.

En la economía en estudio, los partidos tienen preferencias por los instrumentos de política pública. En este contexto se estudia el diseño de impuestos que buscan contener la contaminación para dos casos de interés: 1) la economía tiene un sistema electoral mayoritario, y 2) la economía tiene un sistema electoral de representación proporcional. Estos diferentes sistemas electorales implican que en sistemas electorales mayoritarios existan un menor número de partidos efectivos (véase Liphart, 1994), lo que tiene implicaciones en el número de partidos que cuentan con representación en el Congreso y en la negociación legislativa que determina la política pública. 
El análisis aquí presentado produce varios resultados que son de interés. Primero, en una economía con un sistema electoral mayoritario la política de regulación ambiental es polarizante. Es decir, se implementan impuestos ambientales significativamente mayores o menores al socialmente óptimo dependiendo de la identidad del partido gobernante. En este equilibrio, las políticas de los partidos no convergen (a diferencia de lo que propone el modelo del votante mediano, véase Downs, 1957) lo que logra explicar lo estilizado en economías modernas: los partidos de izquierda y de derecha implementan políticas públicas diferentes.

Segundo, en una economía con un sistema electoral de representación proporcional, la política de equilibrio de regulación ambiental es moderada, es decir, no es una política con un nivel significativamente alto ni bajo de impuestos que buscan reducir la contaminación como ocurriría en el equilibrio de una economía con un sistema electoral mayoritario. Tercero, es más probable que la política pública diseñada en un sistema electoral de representación proporcional se acerque más a la política de regulación ambiental considerada como socialmente deseable.

El artículo se presenta de la siguiente manera: la sección dos contiene una breve revisión de la literatura existente. La sección tres introduce el modelo teórico y muestra la distribución de preferencias por política ambiental de la economía y también se caracteriza la política ambiental socialmente óptima. La sección cuatro contiene el modelo de diseño de política ambiental para una economía con un sistema electoral mayoritario. La sección cinco estudia el caso de un sistema electoral de representación proporcional. La sección seis contiene una discusión de los resultados. Finalmente se presentan las conclusiones en la sección siete.

\section{REVISIÓN DE LA LITERATURA}

Los evidentes problemas relacionados a los efectos negativos de la contaminación han producido un renovado interés por estudiar la relación entre la competencia política y el diseño e implementación de la política ambiental. Para el caso de Estados Unidos, la evidencia sugiere que las políticas ambientales son determinadas por el esfuerzo de cabildeo de grupos de interés (véanse List y Sturm, 2006); mientras que el grado de democracia tiene un efecto positivo en la calidad del aire, los sistemas presidenciales tienen un efecto negativo (Bernauer y Koubi, 2009). 
Los hechos estilizados también sugieren que no sólo las elecciones son importantes determinantes del diseño de política pública sino también la ingeniería del sistema electoral. En particular, la evidencia muestra que el gasto público es mayor en economías con sistemas electorales de representación proporcional que en sistemas mayoritarios (véanse Persson y Tabellini, 2003), y la provisión de bienes públicos en sistemas mayoritarios y de representación proporcional no convergen (véanse Lizzeri y Persico, 2001). En particular, para el caso de bienes públicos locales la evidencia sugiere que las instituciones de los sistemas electorales, en particular, los procesos de nominación de candidatos y la centralización de los partidos, afectan la eficiencia del gasto público local en salud y educación (Hankla et al., 2019).

Investigaciones recientes muestran que las políticas ambientales son más restrictivas en sistemas electorales proporcionales que en sistemas mayoritarios (véanse Fredriksson y Millimet, 2004), y que otro importante determinante en el diseño y eficacia de la regulación ambiental podría ser la estructura de gobierno, es decir, si en la economía existe centralización o descentralización fiscal (véanse Fredriksson et al., 2010). El trabajo de Fredriksson y Millimet (2004) es un análisis empírico que muestra que políticas ambientales son más restrictivas en sistemas electorales proporcionales que en sistemas mayoritarios.

A pesar de las contribuciones de los trabajos antes señalados, algunas de las características de los sistemas electorales (en particular, el número de partidos efectivos y la negociación política en el Congreso) que podrían ser relevantes para el diseño de la política ambiental, no han sido estudiadas adecuadamente. Esa es precisamente la contribución de este trabajo, ya que se analiza el efecto de estas dos instituciones políticas en el diseño de la política ambiental (entendida como los impuestos que buscan reducir el impacto negativo de la contaminación en la sociedad). Además, contribuye al plantear proposiciones teóricas que pueden ser verificadas de manera empírica.

Este trabajo se distingue de la literatura conocida al utilizar una concepción distinta de la competencia electoral. Mientras que la mayoría de la literatura utiliza el modelo de Downs (1957) que asume que los partidos diseñan plataformas económicas sólo para ganar la elección, aquí se utiliza un modelo de competencia electoral a la Wittman (1973) para explicar el diseño de la política pública. Así, se producen predicciones teóricas distintas a las que produce el modelo del votante mediano o modelo de Downs. De tal forma que una contribución del presente trabajo es plantear proposiciones teóricas que pueden ser verificadas empíricamente. 


\section{PREFERENCIAS DE LAS FAMILIAS Y LA POLÍTICA AMBIENTAL SOCIALMENTE ÓPTIMA}

Se asume una economía constituida por $h=1,2 \ldots H$ familias. Las familias en esta economía consumen un solo bien $x_{h}$ que causa una externalidad negativa en el resto de los miembros de la sociedad. Para simplificar el problema de asignación de recursos para la familia $h$ asumimos que la familia $h$ decide el nivel óptimo de $x_{h}$ para maximizar el siguiente problema de asignación de recursos $\mathrm{a}:{ }^{1}$

$$
\operatorname{Max}_{\left\{x_{h}\right\}} \quad v_{h}=\ln \left(x_{h}\right)-\theta_{h} x_{h}-p_{x} x_{h}
$$

Donde $v_{h}=\ln \left(x_{h}\right)-\theta_{h} x_{h}$ representa la estructura de preferencias de la familia tipo $h$ donde $x_{h}$ es el consumo privado del bien $x$ y $\theta_{h}>0$ es una constante. El consumo del bien $x_{h}$ causa una externalidad negativa a todas las familias en la economía, ya que este bien causa contaminación (considere aquellos bienes cuyo consumo induce un efecto residual en la contaminación ambiental). Sin embargo, la familia tipo $h$ sólo considera cómo el consumo de este bien afecta su propio bienestar sin tomar en cuenta la externalidad negativa que su consumo causa en el resto de las familias de la sociedad. El efecto personal de la contaminación del bien $x_{h}$ en la familia tipo $h$ está dado por $-\theta_{h} x_{h}$.

Para simplificar el análisis, se considera que en esta economía existe una oferta perfectamente elástica del bien $x_{h}$ al precio $p_{x}$. En este caso, la posición de la curva de demanda agregada de la economía determina el nivel de equilibrio de consumo en una economía de mercado. Para ver esto, la proposición 1 muestra la demanda óptima del bien $x_{h}$ para la familia tipo $h$ y el nivel agregado del consumo de este bien.

Proposición 1. El nivel de consumo de mercado de la familia tipo $h$ por el bien $x_{h}^{m}$ está dado por: ${ }^{2}$

1 En la literatura es común utilizar funciones indirectas como la que se muestra en la ecuación (1) (véase Besley y Coate, 2003, entre otros).

2 La ecuación (2) se obtiene fácilmente al resolver el problema de asignación de recursos de la familia caracterizado en la ecuación (1). 
$x_{h}^{m}=\frac{1}{p_{x}+\theta_{h}} \quad \forall h=1,2 \ldots . H$

Lo que implica un nivel de equilibrio agregado de mercado para el bien que contamina dado por:

$X^{m}=\sum_{h=1}^{H} x_{h}^{m}=\sum_{h=1}^{H} \frac{1}{p_{x}+\theta_{h}}$

La proposición 1 muestra que las familias seleccionan el nivel de consumo en una economía de mercado determinado por $x_{h}^{m}=\frac{1}{p_{x}+\theta_{h}} \forall h$ lo que implica que las familias toman en cuenta el precio de producción del bien $p_{x}$ y el impacto de la contaminación en la producción del bien $x_{h}$ en su propio bienestar $\theta_{h}$ (incrementos en el precio del bien y en el costo personal de la familia de la contaminación asociada al bien $x_{h}$ reducen la demanda del bien $x_{h}^{m}$ para esta familia). El consumo agregado de este bien en la economía está dado $X^{m}=\sum_{h=1}^{H} x_{h}^{m}=\sum_{h=1}^{H} \frac{1}{p_{x}+\theta_{h}}$ por lo que a nivel agregado la demanda de este bien depende del precio del bien y de la distribución del costo por contaminación que cada una de las familias percibe. Las asignaciones $x_{h}^{m}$ y $X^{m}$, sin embargo, no son Pareto eficiente porque las familias, y el mecanismo de precios del mercado fallan en tomar en cuenta cómo su consumo impacta en el bienestar del resto de las familias en la economía. Es decir, en la decisión de las familias no toman en cuenta el costo marginal social de consumir $x_{h}^{m}$ por lo que el equilibrio de mercado del bien es superior al nivel Pareto eficiente de este bien.

\section{La asignación Pareto eficiente de la economía}

En esta sección se caracteriza la asignación Pareto eficiente del bien $X=\sum_{h=1}^{H} x_{h}$. Para desarrollar el presente análisis se considera una economía controlada por un planeador social benevolente que selecciona el nivel óptimo de consumo para cada una de las familias, y consecuentemente el nivel socialmente óptimo del consumo. La asignación del bien $x_{h}$ para cada una de las familias busca maximizar una función de bienestar social utilitariana simétrica $\Psi$. Es decir, el problema de asignación de recursos del planeador social benevolente es: 
$\operatorname{Max}_{\left\{x_{h}^{*}\right\}} \Psi=\sum_{h=1}^{H} v_{h}=\sum_{h=1}^{H} \ln \left(x_{h}\right)-\sum_{h=1}^{H} \theta_{h} x_{h}-p_{x} \sum_{h=1}^{H} x_{h}$

A continuación, la proposición 2 caracteriza la asignación Pareto eficiente del bien privado de esta economía.

Proposición 2. El nivel de consumo Pareto eficiente para la familia tipo $h$ es $x_{h}^{*}$ que satisface:

$x_{h}^{*}=\frac{1}{p_{x}+\sum_{h=1}^{H} \theta_{h}} \quad \forall h=1,2 \ldots . H$

Lo que implica el nivel de equilibrio agregado Pareto eficiente del bien $X^{*}$ es:

$X^{*}=\sum_{h=1}^{H} x_{h}^{*}=\frac{H}{p_{x}+\sum_{h=1}^{H} \theta_{h}}=\frac{1}{\frac{p_{x}}{H}+E\left[\theta_{h}\right]}$

Donde $E\left[\theta_{h}\right]$ es el costo promedio de la contaminación del bien privado, el cual está dado por:

$E\left[\theta_{h}\right]=\frac{\sum_{h=1}^{H} \theta_{h}}{H}$

La demostración aparece en el Apéndice.

La proposición 2 muestra el nivel Pareto eficiente del consumo del bien privado para las familias tipo $h$ y determinado por $x_{h}^{*}=\frac{1}{p_{x}+\sum_{h=1}^{H} \theta_{h}}$ lo que implica que los determinantes del consumo socialmente óptimo de la familia tipo $h$ es el precio de producción del bien $p_{x}$, el impacto de la contaminación en la producción del bien $x_{h}$ en su propio bienestar $\theta_{h}$ y el impacto de la contaminación o la externalidad negativa del consumo del bien $x$ en la sociedad. La suma del costo personal de la contaminación para la familia $h$ y de la externalidad negativa en la sociedad por su consumo es $\sum_{h=1}^{H} \theta_{h}$. A nivel agregado, el nivel de consumo Pareto eficiente es denotado por $X^{*}$ y representado en la ecuación (6) de la proposición 2. Así, el nivel socialmente óptimo del consumo agregado $X^{*}$ depende negativamente del precio y del efecto de la contaminación negativa en la sociedad. 
La gráfica 1 muestra las diferencias entre el nivel de consumo en una economía de mercado $x_{h}^{m}$ en relación a una economía con una provisión Pareto eficiente $x_{h}^{*}$ para el consumo de la familia $h$.

Gráfica 1. Consumo de mercado y consumo Pareto eficiente de las familias

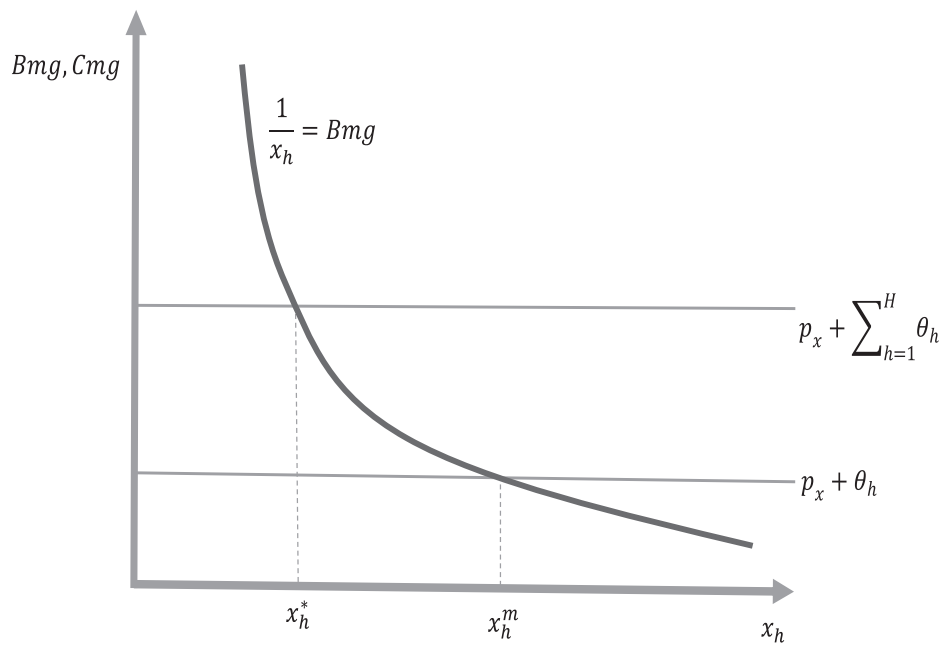

Fuente: elaboración propia.

En la proposición 3 se analiza el tipo de política económica que podría implementar la asignación socialmente óptima de la contaminación. En particular, la proposición 3, muestra que un impuesto Piguviano dado por $\tau_{h}^{*}=\sum_{h=1}^{H} \theta_{h}-\theta_{h}$ puede inducir una asignación Pareto eficiente en una economía de mercado, ya que el impuesto induce a los consumidores a reconocer el costo marginal social de consumir un bien que contamina.

Proposición 3. El nivel de impuestos que induce la asignación Pareto eficiente en una economía de mercado es:

$$
\tau_{h}^{*}=\sum_{h=1}^{H} \theta_{h}-\theta_{h}
$$

Considere ahora el problema de la familia $h$ determinado por: 
$\operatorname{Max}_{\left\{x_{h}^{*}\right\}} v_{h}=\ln \left(x_{h}\right)-\theta_{h} x_{h}-\left\{p_{x}+\tau_{h}^{*}\right\} x_{h}$

Lo que implica que el nivel de consumo de mercado para la familia tipo $h$ dado por $\tilde{x}_{h}^{m}$ tal que

$\tilde{x}_{h}^{m}=\frac{1}{p_{x}+\theta_{h}+\tau_{h}^{*}}=\frac{1}{p_{x}+\sum_{h=1}^{H} \theta_{h}} \quad \forall h=1,2 \ldots . H$

Lo que implica un nivel de equilibrio de mercado dado por:

$$
\tilde{X}^{m}=\sum_{h=1}^{H} \tilde{x}_{h}^{m}=\sum_{h=1}^{H} \frac{1}{p_{x}+\theta_{h}+\tau_{h}^{*}}=\frac{H}{\frac{p_{x}}{H}+E\left[\theta_{h}\right]}
$$

De tal forma que una economía de mercado con un impuesto Piguviano $\tau_{h}^{*}=\sum_{h=1}^{H} \theta_{h}-\theta_{h}$ implica que este equilibrio es Pareto eficiente, ya que

$\tilde{X}^{m}=X^{*}$

La demostración aparece en el Apéndice.

La proposición 3 muestra un resultado bien conocido en la literatura: el gobierno puede intervenir en una economía de mercado al establecer un impuesto Piguviano (que en la economía está dado por $\tau_{h}^{*}=\sum_{h=1}^{H} \theta_{h}-\theta_{h}$ ) el cual induce a las familias a reconocer, a través del mecanismo de precios de mercado, los costos sociales de consumir un bien privado que contamina. Por ello, el consumo de las familias en una economía con intervención del gobierno que establece un impuesto Piguviano induce una asignación Pareto eficiente.

\section{DISEÑO DE POLÍTICA PÚBLICA Y EL NIVEL DE CONTAMINACIÓN EN UN SISTEMA ELECTORAL MAYORITARIO}

A continuación se estudiará el diseño de política ambiental cuando los partidos tienen preferencias sobre política pública (véase Wittman, 1973). En este caso, los partidos no necesariamente diseńan políticas públicas para ganar la elección (véase Downs, 1957) sino buscan ganar la elección porque su interés es maximizar las ganancias asociadas al diseño de política económica, es decir, 
los partidos buscan diseñar política pública para beneficiar a sus seguidores. La distinción principal entre el modelo de Downs y el modelo de Wittman es que, en el primer modelo los partidos diseñan política económica para maximizar la probabilidad del partido de ganar la elección, mientras que en el modelo de Wittman, los partidos seleccionan políticas económicas que maximizan las preferencias de una coalición de individuos al interior del partido (por lo general los individuos que lo controlan).

Aquí se utiliza el modelo de Wittman para proponer un modelo que permita hacer predicciones sobre el diseño de la política ambiental en las democracias modernas. En particular, este trabajo plantea un modelo de competencia electoral que permite entender el diseño de política ambiental en economías con diferentes sistemas electorales. Se busca pues estudiar el diseño de política ambiental en economías con un sistema electoral mayoritario y un sistema electoral de representación proporcional. Estos sistemas electorales cubren la gran mayoría de democracias modernas. Es importante enfatizar que los sistemas electorales representan las instituciones que regulan las elecciones, la creación de partidos y el grado de representación de los partidos en el Congreso. Así, los sistemas electorales crean incentivos en la creación y sostenimiento del número efectivo de partidos en la democracia y en la competencia electoral y legislativa que determina el diseño de política pública (véase Liphart, 1994).

En el caso de estudio, los partidos proponen plataformas políticas en el tema ambiental, los votantes observan las políticas planteadas por los diferentes partidos y votan por el que propone la política económica que más se acerca a sus propias preferencias sobre la política económica que los votantes desean que se implemente. Después de la elección, el partido ganador toma todo (el control del Ejecutivo y del Legislativo) e implementa la política económica que maximiza los intereses o preferencias del partido. En la economía en estudio existen dos partidos denominados por $L$ y $R$ y sus plataformas políticas están caracterizadas por una regulación ambiental determinada por los impuestos $\tau_{L}^{*} \geq 0$ y $\tau_{R}^{*} \geq 0$ que buscan disminuir la contaminación ambiental.

En un extremo, alguno (o ambos) partidos podrían proponer implementar un impuesto igual a cero lo que implicaría una política ambiental por completo flexible donde la economía de mercado opera sin intervención del Estado, y en el otro extremo, alguno (o ambos) partidos podrían proponer una regulación ambiental con el máximo impuesto permisible en la economía induciendo también un nivel socialmente subóptimo del nivel de contaminación. En este último caso, el costo en la asignación de recursos para la sociedad es una reducción masiva de bienes privados que contaminan. 
Así, en esta economía, el diseño de política ambiental se estudia a través de un juego dinámico de información perfecta donde el equilibrio político involucra las siguientes etapas:

Primera Etapa: los partidos seleccionan una plataforma política en materia ambiental $\tau_{L}^{*} \geq 0$ y $\tau_{R}^{*} \geq 0$.

Segunda Etapa: los individuos observan las políticas de ambos partidos y su voto es secuencialmente racional, es decir, los votantes votan, no en función de las plataformas de los partidos sino en función del tipo de políticas que cada uno implementaría una vez que el partido que resulte ganador en las elecciones forme el gobierno. El cálculo de bienestar de un votante tipo $\theta_{h}$ está determinado por $\chi_{L}\left(\theta_{h}\right)$ cuya función está dada por $\chi_{L}\left(\theta_{h}\right)=v_{L}\left(\tau_{L}^{*}, \theta_{h}\right)-v_{R}\left(\tau_{R}^{*}, \theta_{h}\right)$, donde $v_{L}\left(\tau_{L}^{*}, \theta_{h}\right)=\ln \left(x_{h}\right)-\theta_{h} x_{h}-\left\{p_{x}+\tau_{L}^{*}\right\} x_{h}$ y $v_{R}\left(\tau_{R}^{*}, \theta_{h}\right)=\ln \left(x_{h}\right)-\theta_{h} x_{h}$ $-\left\{p_{x}+\tau_{R}^{*}\right\} x_{h}$. Si $\chi_{L}\left(\theta_{h}\right)>0$ el votante tipo $\theta_{h}$ vota por el partido $L$ y si $\chi_{L}\left(\theta_{h}\right)<0$, el individuo vota por el partido $R$.

Tercera Etapa: el partido que es electo implementa la política que maximiza las preferencias del individuo representativo al interior del partido.

Las preferencias de los partidos $Z=L, R$ están dadas por $\mu_{z}=\ln \left(x_{z}\right)-\theta_{z} x_{z}$ donde $x_{z}$ es un bien consumido por el individuo representativo del partido $Z$ y $\theta_{z}>0$ es una constante. El efecto de la contaminación del bien $x_{z}$ en la familia que controla el partido $Z$ está dado por $-\theta_{z} x_{z}$. La política ambiental está caracterizada por un impuesto $\tau_{z}^{*} \geq 0 \forall Z=L, R$. Así, el problema de diseño de política ambiental es establecer el nivel del impuesto $\tau_{z}^{*} \geq 0 \forall Z=L, R$ que maximiza las preferencias del partido dadas por:

$v_{z}=\ln \left(x_{z}\right)-\theta_{z} x_{z}-\left\{p_{x}+\tau_{z}\right\} x_{z} \quad \forall Z=L, R$

Para esta economía, el equilibrio político-económico se caracteriza por el equilibrio perfecto de Nash del subjuego como se muestra en la definición 1. Sin pérdida de la generalidad del análisis se asume $\theta_{L}>E\left[\theta_{h}\right]>\theta_{R}$ donde $E\left[\theta_{h}\right]=\frac{\sum_{h=1}^{H} \theta_{h}}{H}$. Es decir, el costo de la contaminación es mayor para la familia representada por el partido $L$ que el costo promedio de la economía y que su correspondiente costo ambiental para la familia representada por el partido $R$. 
En lo que sigue, la definición 1 caracteriza el equilibrio de Nash perfecto del subjuego (para una caracterización del juego en forma extensiva véase gráfica A.1 del Apéndice).

Definición 1. El equilibrio electoral para una economía con un sistema electoral mayoritario con partidos con preferencias sobre política ambiental está caracterizado de la siguiente manera:

i) En la primera etapa del juego, los partidos proponen $\tau_{z}^{*}=\theta_{z} \forall Z=L, R$ tal que

$\tau_{z}^{*} \in \operatorname{ArgMax} \quad v_{z}=\ln \left(x_{z}\right)-\theta_{z} x_{z}-\left\{p_{x}+\tau_{z}^{*}\right\} x_{z} \quad \forall Z=L, R$

ii) En la segunda etapa, el votante tipo $\theta_{h}$ vota por el partido $L$ si

$\chi_{L}\left(\theta_{h}\right)=v_{L}\left(\tau_{L}^{*}, \theta_{h}\right)-v_{R}\left(\tau_{R}^{*}, \theta_{h}\right)>0$

Si $\chi^{L}\left(\theta_{h}\right)<0$, el individuo vota por el partido $R$

iii) En el tercer escenario, el partido electo implementa $\tau_{z}^{*}=\theta_{z} \forall Z=L, R$ tal que

$\tau_{z}^{*} \in \operatorname{ArgMax} v_{z}=\ln \left(x_{z}\right)-\theta_{z} x_{z}-\left\{p_{x}+\tau_{z}^{*}\right\} x_{z} \quad \forall Z=L, R$

A partir de la definición 1, es fácil observar que la heterogeneidad de las preferencias de los partidos $L$ y $R$ necesariamente implica que en el último escenario del juego las políticas ambientales de los partidos divergen, es decir, $\tau_{L}^{*} \neq \tau_{R}^{*}$.

A continuación, en la proposición 4, se caracteriza el nivel de política ambiental óptima de los partidos $Z=L, R$. La solución que se plantea en la proposición 4 representa las estrategias débilmente dominantes de los partidos $Z=L, R$ en la tercera etapa del juego político de la economía.

Proposición 4. El nivel de consumo de mercado ideal de la familia representativa del partido $Z$ está determinada por $x_{z}$ tal que

$x_{z}=\frac{1}{p_{x}+\theta_{Z}} \quad \forall Z=L, R$

Por lo que el nivel de la política ambiental deseada por el partido $Z$ es: 
$\tau_{Z}^{*}=\theta_{Z} \quad \forall Z=L, R$

Lo que implica un nivel de equilibrio de mercado dado por:

$X^{m}\left(p_{x}, \tau_{z}^{*}\right)=\sum_{h=1}^{H} x_{z}=\sum_{h=1}^{H} \frac{1}{p_{x}+\tau^{* Z}}=\frac{H}{p_{x}+\theta_{Z}} \quad \forall Z=L, R$

La proposición 4 dice que los partidos tienen incentivos electorales a internalizar los efectos negativos de la contaminación sólo en los individuos representados por el partido. Es decir, los partidos en este equilibrio no tienen incentivos a reconocer cómo la contaminación afecta a los individuos que no son representados por el partido. Por ello, el nivel del impuesto Piguviano es $\tau_{z}^{*}=\theta_{Z} \quad \forall Z=L, R$. Correspondiente a este tipo de politica pública, el nivel de consumo de equilibrio en esta economía está dado por $X^{m}\left(p_{x}, \tau_{z}^{*}\right)=\frac{H}{p_{x}+\theta_{Z}} \quad \forall Z=L, R$.

Un resultado interesante de este equilibrio, que es mostrado por la proposición 5, es que el esfuerzo del gobierno de regulación o control de la contaminación depende del efecto negativo de la contaminación en los individuos que el partido representa. Dado que en nuestra economía $\theta_{L}>E\left[\theta_{h}\right]>\theta_{R}$, entonces pueden existir equilibrios con un alto compromiso del gobierno por controlar la contaminación que corresponde a un valor elevado de $\tau_{L}^{*}=\theta_{L}$. A la vez, pueden existir equilibrios con un bajo compromiso del gobierno por controlar la contaminación que corresponde a un valor bajo de $\tau_{R}^{*}=\theta_{R}$. Este resultado se explica debido a la heterogeneidad de preferencias de las familias que integran los partidos determina la heterogeneidad de las políticas ambientales que son consideradas ideales por los partidos.

Proposición 5. La heterogeneidad de las preferencias de las familias representativas al interior del partido determina la política ambiental a ser implementada por cada partido, tal que

$$
\begin{gathered}
\tau_{R}^{*}=\theta_{R} \\
y \\
\tau_{L}^{*}=\theta_{L}
\end{gathered}
$$


De tal forma que $\theta_{L}>E\left[\theta_{h}\right]>\theta_{R}$ implica

$\tau_{R}^{*}<\tau_{L}^{*}$

A continuación, la proposición 6 muestra que si el partido $R$ gana el control del gobierno (al tomar todo y controlar el Poder Ejecutivo y Legislativo) el nivel de contaminación agregada en la economía será superior al socialmente deseable y la proposición 7 muestra que si el partido $L$ gana la elección el nivel de contaminación agregada en la economía será inferior al socialmente deseable.

Proposición 6. Si una mayoría vota por el partido de derecha entonces $\tau_{R}^{*}=\theta_{R}$ y $\theta_{L}>E\left[\theta_{h}\right]>\theta_{R}$ implican que en una economía con un sistema electoral mayoritario puede producir demasiada contaminación, ya que

$X^{m}\left(p_{x}, \tau_{R}^{*}\right)>X^{*}$

\section{Demostración}

Por las condiciones de equilibrio caracterizadas en las proposiciones $2 \mathrm{y}$ 4 se satisface que $X^{m}\left(p_{x}, \tau_{R}^{*}\right)=\frac{1}{\frac{p_{x}}{H}+\frac{\theta_{R}}{H}}$ mientras que $X^{*}=\sum_{h=1}^{H} x_{h}^{*}=\frac{1}{\frac{p_{X}}{H}+E\left[\theta_{h}\right]}$ donde $E\left[\theta_{h}\right]=\frac{\sum_{h=1}^{H} \theta_{h}}{H}$. Por lo tanto $\theta_{L}>E\left[\theta_{h}\right]>\theta_{R}$ implica $X^{m}\left(p_{x}, \tau_{R}^{*}\right)>X^{*}$.

Proposición 7. Si una mayoría vota por el partido de izquierda entonces $\tau^{* L}=\theta_{L}$ y $\theta_{L}>E\left[\theta_{h}\right]>\theta_{R}$ implican que en una economía con un sistema electoral mayoritario puede producir un nivel de contaminación inferior a la contaminación socialmente deseable.

$X^{m}\left(p_{x}, \tau_{L}^{*}\right)<X^{*}$

\section{Demostración}

Este resultado sigue trivialmente a partir de la proposición 6 .

Las proposiciones 6 y 7 muestran condiciones en las que en una economía con un sistema electoral mayoritario el gobierno puede implementar una política ambiental que acepta o tolera un alto nivel de contaminación y también identifica condiciones en las que el gobierno establece una sobre regulación ambiental que conduce a un nivel de contaminación menor al socialmente de- 
seable $X^{*}$, la cual es una asignación Pareto eficiente de la economía. La razón por la que un gobierno podría no desear combatir la contaminación es que el partido en el gobierno puede estar representado por individuos con un mayor beneficio neto del consumo del bien que contamina (es decir, el gobierno podría estar controlado por un partido a su vez controlado por individuos con un nivel muy bajo del parámetro $\theta_{R}$ ) y, por lo tanto, un gobierno controlado por este tipo de coalición de votantes seleccionará una política ambiental que conduce a una alta contaminación.

En contraparte, un gobierno podría desear reducir significativamente la contaminación si está representado por individuos que se ven perjudicados de una forma significativa por el consumo del bien que contamina (es el caso en el que el gobierno está controlado por individuos con un nivel alto del parámetro $\left.\theta_{L}\right)$ y, por lo tanto, un gobierno controlado por este tipo de coalición de votantes seleccionará una política ambiental restrictiva que conduzca a una baja contaminación (véase gráfica 2).

Es importante comentar que, en un sistema electoral mayoritario, el votante mediano es el votante decisivo para otorgar mayorías relativas a alguno de los partidos. Por ello, el proceso electoral, a través del votante mediano, determina cuál de los partidos gana la elección con al menos una mayoría de los votos y controla el diseño de la política ambiental.

Gráfica 2. Políitca ambiental en un sistema electoral mayoritario

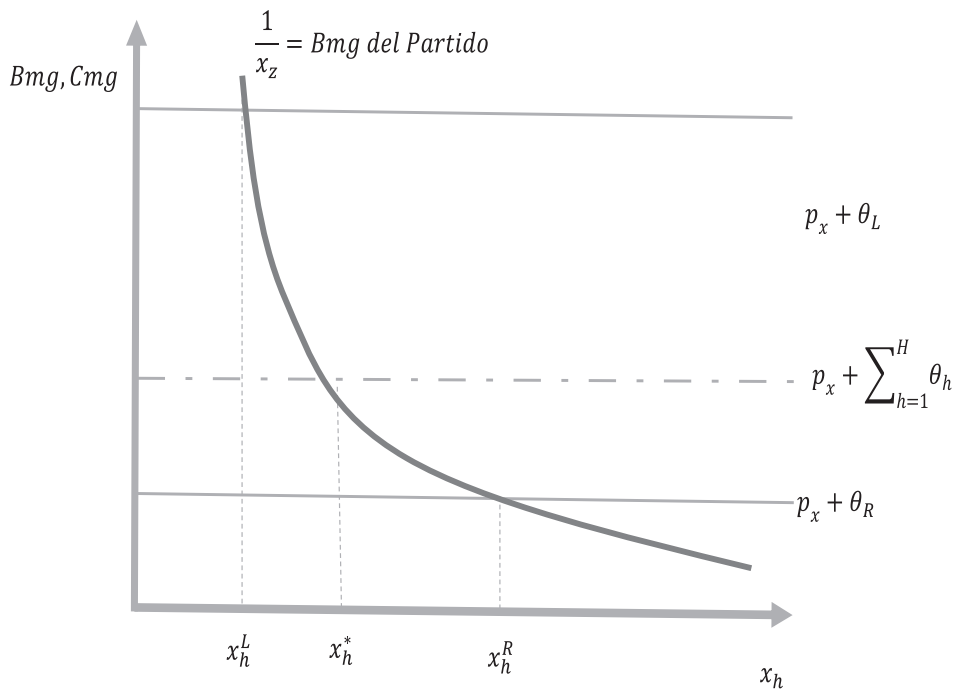

Fuente: elaboración propia. 


\section{REGULACIÓN AMBIENTAL EN UN SISTEMA DE REPRESENTACIÓN PROPORCIONAL}

A continuación se presenta el desarrollo de un modelo con una economía con un sistema electoral de representación proporcional. Una característica distintiva de estos sistemas electorales es que promueven la existencia de más de dos partidos (debido a que las condiciones de registro de partido son más flexibles en los sistemas electorales de representación proporcional que en los sistemas electorales mayoritarios). Por lo tanto, la consideración de un sistema electoral de representación proporcional implica la competencia electoral entre más de dos partidos, a diferencia del sistema mayoritario donde los partidos efectivos pueden llegar a ser dos (véase Liphart, 1994).

Otra diferencia importante asociada con la competencia electoral con partidos múltiples es que, en sistemas de representación proporcional, es menos probable que un solo partido controle el Poder Legislativo. Es pues, poco probable que el partido con una pluralidad relativa de los votos en la elección del Poder Ejecutivo tenga, además, una mayoría absoluta en el Congreso para establecer políticas públicas. En contraste, en el caso de un sistema electoral mayoritario, es más probable que el partido que gana las elecciones también pueda tener una mayoría absoluta en el Congreso. Sin embargo, en el caso de una economía con un sistema electoral proporcional (debido a que el voto se distribuye entre más partidos), es probable que un partido tenga que negociar con el resto de los partidos en el Congreso el diseño e implementación de política pública.

El análisis de esta sección incorpora estas dos diferencias de los sistemas electorales mayoritarios y proporcionales: primero, el número de partidos efectivos es mayor en sistemas proporcionales y, por ello, en el modelo empleado se considera la competencia electoral de dos partidos en un sistema electoral mayoritario y de tres partidos en un sistema de representación proporcional y, segundo, que en un sistema de representación proporcional, el Poder Ejecutivo tiene que negociar con el Poder Legislativo el diseño e implementación de política pública. Por ello, se incorpora explícitamente un juego con un proceso electoral al que le sigue un juego de negociación legislativa en el Congreso. ${ }^{3}$

3 En la sección anterior, y siguiendo la literatura, se asume que, en una economía con un sistema electoral mayoritario, el partido que gana la elección "toma todo". Este supuesto significa que el partido que gana la elección conforma el Poder Ejecutivo y tiene una mayoría absoluta en el Congreso. 
Para considerar el caso de la competencia electoral entre multi partidos, se planteó un modelo dinámico de información perfecta de cuatro etapas. En la primera los partidos proponen sus plataformas de política ambiental. En la segunda, los votantes observan las plataformas de los partidos y votan por la opción que más se acerque a la política ambiental ideal del votante. En la tercera, la elección toma lugar y los partidos reciben una proporción de asientos en el Poder Legislativo equivalente a la proporción de votos recibidos en la elección. En la cuarta etapa, la negociación legislativa toma lugar. El proceso de diseño de política es el siguiente: si un partido tiene al menos una mayoría simple en el Congreso, entonces el partido propone la política ambiental que maximiza las preferencias del partido. Si ningún partido tiene una mayoría de los asientos en el Congreso, entonces cada uno de los partidos hace una propuesta de política ambiental. Todas las propuestas se comparan en el Congreso y la propuesta que recibe una mayoría de los votos es implementada en el Congreso. ${ }^{4}$ Debido a que las políticas públicas satisfacen el requerimiento de preferencias de un solo pico (en la literatura se conoce como single peaked preferences), entonces la política ideal del votante mediano es la ganadora de la ronda de comparaciones entre las diferentes opciones de política pública (para una demostración de este resultado véase Bowen, 1943).

En esta economía, se asume que existen tres partidos definidos por $Z=L, M, R$ donde el partido $L$ es un partido que lo denominaremos de izquierda y el partido $R$ que se denominará como un partido de derecha y $M$ es el partido de centro.

El gobierno interviene en esta economía y el partido $Z$ propone un impuesto Piguviano $\tau_{z} \forall Z=L, M, R$ como un mecanismo para internalizar los costos causados por la contaminación. Las preferencias del partido son definidas a partir de las preferencias de individuos que controlan el partido (como se definió en la sección anterior). Así, el problema de asignación de recursos de una familia representativa del partido $Z$ es el siguiente:

$\operatorname{Max}_{\left\{x_{z}^{*}\right\}} \quad v_{z}=\ln \left(x_{z}\right)-\theta_{z} x_{z}-\left\{p_{x}+\tau_{z}\right\} x_{z} \quad \forall Z=L, M, R$

Como se muestra en la definición 2, para esta economía, el equilibrio político-económico se caracteriza por el equilibrio perfecto de Nash del subjuego.

4 La comparación de las alternativas de política pública se hace a través de rondas de votos donde la alternativa uno se compara contra la alternativa dos, la ganadora de estas opciones se compara con la tercera alternativa, etcétera. Hasta que todas las posibles opciones han sido votadas. 
Sin pérdida general del análisis se asume $\theta_{L}>\theta_{M}>\theta_{R}$ (para una caracterización del juego en forma extensiva, véase gráfica A.2 del Apéndice).

Definición 2. El equilibrio electoral para una economía con un sistema electoral proporcional con partidos $Z=L, M, R$ con preferencias sobre la política ambiental está caracterizado de la siguiente manera:

i) En la primera etapa del juego, los partidos proponen $\tau_{z}^{*}=\theta_{z} \forall Z=L, M, R$ tal que

$\tau_{z}^{*} \in \operatorname{ArgMax} v_{z}=\ln \left(x_{z}\right)-\theta_{z} x_{z}-\left\{p_{x}+\tau_{z}\right\} x_{z} \quad \forall Z=L, M, R$

ii) En la segunda etapa del juego, el votante tipo $\theta_{h}$ vota

Por el partido $L$ si $\chi_{L R}\left(\theta_{h}\right)=v_{L}\left(\tau_{L}^{*}, \theta_{h}\right)-v_{R}\left(\tau_{R}^{*}, \theta_{h}\right)>0$

у $\chi_{L M}\left(\theta_{h}\right)=v_{L}\left(\tau_{L}^{*}, \theta_{h}\right)-v_{M}\left(\tau_{M}^{*}, \theta_{h}\right)>0$

Por el partido $M$ si $\chi_{M R}\left(\theta_{h}\right)=v_{M}\left(\tau_{M}^{*}, \theta_{h}\right)-v_{R}\left(\tau_{R}^{*}, \theta_{h}\right)>0$

у $\chi_{M L}\left(\theta_{h}\right)=v_{M}\left(\tau_{M}^{*}, \theta_{h}\right)-v_{L}\left(\tau_{L}^{*}, \theta_{h}\right)>0$

Por el partido $R$ si $\chi_{R M}\left(\theta_{h}\right)=v_{R}\left(\tau_{R}^{*}, \theta_{h}\right)-v_{M}\left(\tau_{M}^{*}, \theta_{h}\right)>0$

у $\chi_{R L}\left(\theta_{h}\right)=v_{R}\left(\tau_{R}^{*}, \theta_{h}\right)-v_{L}\left(\tau_{L}^{*}, \theta_{h}\right)>0$

iii) En la tercera etapa del juego, cada uno de los partidos recibe una proporción $\Omega_{z}$ de los asientos del Congreso tal que

$\Omega_{L}, \Omega_{M}, \Omega_{R} \in[0,1]: \Omega_{L}+\Omega_{M}+\Omega_{R}=1$

iv) En la cuarta etapa del juego:

a) $\mathrm{Si} \Omega_{z} \geq \frac{1}{2}$ entonces el partido $Z$ tiene una mayoría en el Congreso por lo que ese partido implementa $\tau_{z}^{*}=\theta_{z}$ tal que

$\tau_{z}^{*} \in \operatorname{ArgMax} v_{z}=\ln \left(x_{z}\right)-\theta_{z} x_{z}-\left\{p_{x}+\tau_{z}\right\} x_{z} \quad \forall Z=L, M, R$ 
b) De lo contrario, si $\Omega_{Z}<\frac{1}{2} \forall Z=L, M, R$ entonces ningún partido tiene una mayoría por lo que se comparan las tres alternativas de política ambiental $\tau_{L}^{*}, \tau_{M}^{*}, \tau_{R}^{*}$ y se implementa la política que tiene la mayoría en el Congreso.

A continuación, en la proposición 8 , se caracteriza el nivel de política ambiental óptima de los partidos $Z=L, M, R$. La solución que se plantea en la proposición 8 representa las estrategias débilmente dominantes de los partidos $Z=L, M, R$ en la cuarta etapa del juego.

Proposición 8. El nivel de consumo de mercado ideal de la familia representativa del partido $Z$ está determinado por $x_{h}^{Z}$ tal que

$$
x_{z}=\frac{1}{p_{x}+\theta_{z}} \quad \forall Z=L, M, R
$$

Por lo que el nivel de la política ambiental deseada por el partido $Z$ es:

$$
\tau_{z}^{*}=\theta_{Z} \quad \forall Z=L, M, R
$$

Lo que implica un nivel de equilibrio de mercado dado por:

$$
X^{m}\left(p_{x}, \tau_{Z}^{*}\right)=\sum_{h=1}^{H} x_{h}^{m}=\sum_{h=1}^{H} \frac{1}{p_{x}+\tau_{z}^{*}}=\frac{1}{\frac{p_{x}}{H}+\frac{\theta_{Z}}{H}} \quad \forall Z=L, M, R
$$

De una forma similar al equilibrio económico-político de la sección anterior, la proposición 8 dice que los partidos tienen incentivos electorales a internalizar los efectos negativos de la contaminación sólo en los individuos representados por el partido. Es decir, los partidos no tienen incentivos a reconocer cómo la contaminación afecta a los individuos que no forman parte del partido. Por ello, el nivel del impuesto Piguviano es $\tau_{z}^{*}=\theta_{Z} \quad \forall Z=L, M, R$. Correspondiente a este tipo de política pública, el nivel de consumo agregado de equilibrio en esta economía está dado por $X^{m}\left(p_{x}, \tau_{z}^{*}\right)=\frac{1}{\frac{p_{x}}{H}+\frac{\theta_{Z}}{H}} \quad \forall Z=L, M, R$.

Un resultado interesante de este equilibrio es que el esfuerzo del gobierno por regular la contaminación depende del efecto de ésta en los individuos que el partido representa. Por ello, para esta economía pueden existir equi- 
librios con un alto compromiso del gobierno por regular la contaminación que corresponde a un valor elevado de $\tau_{L}^{*}=\theta_{L}$. A la vez, pueden existir equilibrios con un bajo compromiso del gobierno por regular la contaminación que corresponde a un valor bajo de $\tau_{R}^{*}=\theta_{R}$. El nivel intermedio de regulación ambiental, en relación a los dos extremos antes planteados, está dado por un impuesto determinado por $\tau_{M}^{*}=\theta_{M}$. Este resultado, en particular, se caracteriza en la proposición 9 que muestra que la heterogeneidad de preferencias de las familias que integran los partidos determina la heterogeneidad de las políticas ambientales que son consideradas ideales por los partidos.

Proposición 9. La heterogeneidad de las preferencias de las familias representativas al interior del partido determinada política ambiental a ser implementada por cada partido tal que

$$
\begin{aligned}
& \tau_{R}^{*}=\theta_{R} \\
& \tau_{M}^{*}=\theta_{M} \\
& \tau_{L}^{*}=\theta_{L}
\end{aligned}
$$

De tal forma que $\theta_{L}>\theta_{M}>\theta_{R}$ implica

$$
\tau_{R}^{*}<\tau_{M}^{*}<\tau_{L}^{*}
$$

Con más de dos partidos, el juego político por el voto de la población cambia significativamente. Si alguno de los partidos tiene al menos una mayoría de los votos en la elección, entonces esa política se implementa en el Congreso. Si ninguno de los partidos tiene una mayoría en el Congreso, entonces de las posibles políticas a votar en la negociación legislativa, es decir, $\tau_{R}^{*}=\theta_{R}, \tau_{M}^{*}=\theta_{M}, \tau_{L}^{*}=\theta_{L}$, la política $\tau_{M}^{*}=\theta_{M}$ es la que recibe una mayoría de votos en un proceso de negociación legislativa. Para ver esto, note que las preferencias de los partidos satisfacen la propiedad de un solo pico (en la literatura esto se conoce como singled peaked preferences). Así, si ninguno de los partidos tiene una mayoría, entonces la política del partido $\mathrm{M}$ es la política mediana de las tres alternativas y, por lo tanto, recibe la mayoría de los votos en la negociación legislativa. Este resultado se caracteriza formalmente en la proposición 10. 
Proposición 10. Si $\Omega_{z} \geq \frac{1}{2}$ para algún partido $z$, entonces este partido tiene una mayoría en el Congreso por lo que ese partido implementa $\tau_{z}^{*}=\theta_{z} \quad \forall Z=L, M, R$ tal que

$\tau_{z}^{*} \in \operatorname{ArgMax} \quad v_{z}=\ln \left(x_{z}\right)-\theta_{z} x_{z}-\left\{p_{x}+\tau_{z}\right\} x_{z} \quad \forall Z=L, M, R$

De lo contrario, si $\Omega_{z}<\frac{1}{2} \forall Z$ entonces ningún partido z tiene una mayoría por lo que se comparan las tres alternativas de política ambiental $\tau_{L}^{*}, \tau_{M}^{*}, \tau_{R}^{*}$ y la política mediana del proceso legislativo es $\tau_{M}^{*}=\theta_{M}$.

La principal implicación de la proposición 10 es que, en ausencia de que algún partido obtenga la mayoría absoluta en la elección, entonces la política ambiental que se espera se implemente en una economía con un sistema electoral de representación proporcional es $\tau_{M}^{*}=\theta_{M}$ y que es considerada una política ambiental mediana o política ambiental moderada a partir de las alternativas existentes. En contraparte, en una economía con un sistema electoral mayoritario las políticas que podrían implementarse son consideradas polarizantes o extremas, es decir, las políticas en el equilibrio podrían ser $\tau_{L}^{*}=\theta_{L} \mathrm{o}$ $\tau_{R}^{*}=\theta_{R}$.

Por lo tanto, en una economía con un sistema electoral mayoritario el gobierno puede implementar una política ambiental que acepta o tolera un alto nivel de contaminación, $\tau_{R}^{*}=\theta_{R}$ o también identifica condiciones en las que el gobierno establece una sobre regulación ambiental que conduce a un nivel de contaminación menor al socialmente deseable $X^{*}$, es decir, $\tau_{L}^{*}=\theta_{L}$. En cambio, la negociación legislativa de los sistemas electorales de representación proporcional induce la política del votante mediano del Congreso que en este caso es la política de regulación moderada de las tres alternativas en nuestra economía, es decir, la política a implementarse es $\tau_{M}^{*}=\theta_{M}$.

\section{DISCUSIÓN DE LOS RESULTADOS}

A continuación se presenta un resumen de los resultados del presente trabajo. $\mathrm{El}$ análisis teórico produce varios resultados de interés. Primero, en una economía con un sistema electoral mayoritario la política de regulación ambiental es probable que sea polarizante. Es decir, los impuestos que buscan aminorar los efectos negativos de la contaminación serán diferentes a los impuestos socialmente óptimos. Esto es así, ya que las instituciones políticas de un sistema electoral mayoritario inducen a un equilibrio en el que los partidos implementan políticas de control ambiental significativamente mayores o menores al 
socialmente óptimo dependiendo de la identidad del partido gobernante. Es decir, en este equilibrio, las políticas de los partidos no convergen (a diferencia de lo que propone el modelo del votante mediano en un sistema electoral mayoritario, véase Downs, 1957) lo que logra explicar los hechos estilizados de economías modernas donde los partidos de izquierda y de derecha implementan políticas públicas diferenciadas.

En particular, el modelo predice que si el partido de izquierda representa a votantes que (relativamente hablando) tienen un costo más elevado por la contaminación que los votantes representados por un partido de derecha, entonces, la regulación ambiental propuesta por partidos de derecha será más flexible (tolerará más la contaminación) que la correspondiente política propuesta por un partido de izquierda. Sin embargo, en ninguno de los casos, la política ambiental es socialmente óptima a menos que el partido que gane las elecciones represente al votante promedio del electorado. ${ }^{5}$

Segundo, en una economía con un sistema electoral de representación proporcional, la política de equilibrio de regulación ambiental es moderada, es decir, no es una política con un nivel significativamente alto ni bajo de impuestos que buscan regular el nivel de contaminación como ocurriría en el equilibrio de una economía con un sistema electoral mayoritario. Esto hace más probable que este tipo de políticas adoptadas en una economía con un sistema electoral de representación proporcional, se acerquen más a la política ambiental socialmente deseable.

Esta diferencia importante en el tipo de políticas que se implementarían en las economías con sistemas electorales de representación proporcional y mayoritario se debe a dos razones: i) en un sistema de representación proporcional, las cuotas de registro de partidos son inferiores a las que existen en un sistema mayoritario lo que induce a que existan más partidos efectivos en un sistema de representación proporcional que en el mayoritario (véase a Liphart, 1994). Mientras que, ii) la existencia de más partidos efectivos en un sistema de representación proporcional hace menos probable que un partido tenga el control de los poderes Ejecutivo y Legislativo (aunque esto es posible y de hecho ocurre ocasionalmente en la realidad) y, por lo tanto, el partido en el poder diseñe políticas públicas sin la necesidad de negociar con otros partidos. Es decir, en una economía con un sistema electoral de representación proporcional la competencia electoral en el Poder Legislativo tiende a forzar a

5 Es bien conocido en la literatura que los partidos diseñan políticas ambientales que maximizan el bienestar del votante promedio entonces estas políticas también maximizan el bienestar de la sociedad. 
los partidos a negociar una política ambiental más moderada, mientras que en un sistema electoral mayoritario es más probable que un solo partido tenga el control de los poderes Ejecutivo y Legislativo, en cuyo caso, ese partido en el poder no es forzado a negociar la política ambiental con otros partidos.

En el caso de un sistema electoral mayoritario, la política ambiental refleja los intereses sobre la política pública de una coalición minoritaria lo cual se traduce en políticas públicas polarizantes (que reflejan los intereses extremos de los votantes en el electorado), mientras que un sistema de representación proporcional, la negociación legislativa ayuda a mejorar la representación de los intereses de por lo menos una mayoría, lo que induce a la implementación de políticas públicas moderadas.

\section{CONCLUSIONES}

En el presente análisis se abordó el impacto de las instituciones de los sistemas electorales en el diseño de la política pública, en particular, en el diseño de una política ambiental. Las instituciones del sistema electoral de una democracia podrían estar vinculadas a las políticas ambientales adoptadas en una democracia, ya que estas instituciones determinan el número de partidos que compiten en una elección, así como el nivel de representación de cada uno de los partidos en el Congreso. En este artículo, se desarrolló un modelo de competencia electoral que explica el diseño de impuestos que buscan aminorar los efectos negativos de la contaminación para dos casos de interés: 1) la economía tiene un sistema electoral mayoritario, y 2) la economía tiene un sistema electoral de representación proporcional.

La evidencia empírica muestra que en sistemas electorales mayoritarios existen un menor número de partidos efectivos, lo que tiene implicaciones en la negociación legislativa de los partidos que tienen representación en el Congreso por lo que el control de un solo partido del Congreso es más probable en un sistema electoral mayoritario (en el caso en que un partido gana todo y obtiene el control del Poder Ejecutivo y el Poder Legislativo, dándole amplias posibilidades de diseñar las políticas económicas que desee). En contraparte, en un sistema de representación proporcional existen más partidos efectivos y es más probable que la política pública en el Congreso sea de consenso entre los diferentes partidos.

También se obtuvieron varios resultados teóricos de interés. Primero, en una economía con un sistema electoral mayoritario, la política de regulación ambiental es altamente probable que sea polarizante. Es decir, se implemen- 
tan impuestos ambientales significativamente mayores o menores al socialmente óptimo dependiendo de la identidad del partido gobernante. En este equilibrio las políticas de los partidos no convergen, lo que explica el hecho estilizado que partidos de izquierda y de derecha implementan políticas públicas diferenciadas. Segundo, en una economía con un sistema electoral de representación proporcional, la política de equilibrio de regulación ambiental es moderada (no es una política con un nivel significativamente alto ni bajo de impuestos como ocurriría en el equilibrio de una economía con un sistema electoral mayoritario). Tercero, el análisis predice que las políticas de un sistema electoral de representación proporcional se acercan más a la política de regulación ambiental socialmente deseable.

\section{BIBLIOGRAFÍA}

Bernauer, T. y Koubi, V. (2009), "Effects of political institutions on air quality”, Ecological Economics, vol. 68, núm. 5, Estados Unidos, International Society for Ecological Economics, marzo.

Besley, T. y Coate, S. (2003), "Centralized versus decentralized provision of local public goods: A political economy approach", Journal of Public Economics, vol. 87, núm. 12, Holanda, Elsevier, diciembre.

Bowen, H. R. (1943), "The interpretation of voting in the allocation of economic resources", The Quarterly Journal of Economics, vol. 58, núm. 1, Estados Unidos, Harvard University-Oxford University Press, diciembre.

Downs, A. (1957), An economic theory of democracy, New York, Harper and Brothers.

Fredriksson, P. G. y Millimet, D. L. (2004), "Comparative politics and environmental taxation", Journal of Environmental Economics and Management, vol. 48, núm. 1, Holanda, Elsevier, julio.

, Matschkeb, X. y Minierc, J. (2010), "Environmental policy in majoritarian systems", Journal of Environmental Economics and Management, vol. 59, núm. 2, Holanda, Elsevier, marzo.

Hankla, C., Martínez-Velázquez, J. y Ponce-Rodríguez, R. A. (2019), Local accountability and national coordination in fiscal federalism: A fine balance, Reino Unido, Edward-Elgard Publishing.

Liphart, A. (1994), Electoral systems and party systems: A study of twenty-seven democracies, 1945-1990, Reino Unido, Oxford University Press.

List, J. A. y Sturm, D. M. (2006), "How elections matter: Theory and evidence from environmental policy", The Quarterly Journal Of Economics, 
vol. 121, núm. 4, Estados Unidos, Harvard University-Oxford University Press, noviembre.

Lizzeri, A. y Persico, N. (2001), "The provision of public projects under alternative electoral incentives", American Economic Review, vol. 91, núm. 1, Estados Unidos, American Economic Association, marzo.

Mueller, D. C. (2003), Public choice III, Reino Unido, Cambridge University Press.

Persson, T. y Tabellini, G. (2003), The economic effect of constitutions, Estados Unidos, MIT press.

Wittman, D. A. (1973), "Parties as utility maximizers", American Political Science Review, vol. 67, núm. 2, Estados Unidos, American Political Science Association, junio.

\section{APÉNDICE}

Proposición 2. El nivel de consumo Pareto eficiente para la familia tipo $h$ es $x_{h}^{*}$ que satisface:

$$
x_{h}^{*}=\frac{1}{p_{x}+\sum_{h=1}^{H} \theta_{h}} \quad \forall h=1,2 \ldots . H
$$

Lo que implica el nivel de equilibrio agregado Pareto eficiente del bien $X^{*}$ es:

$$
X^{*}=\sum_{h=1}^{H} x_{h}^{*}=\frac{H}{p_{x}+\sum_{h=1}^{H} \theta_{h}}=\frac{1}{\frac{p_{x}}{H}+E\left[\theta_{h}\right]}
$$

Donde $E\left[\theta_{h}\right]$ es el costo personal promedio de la contaminación del bien privado, el cual está dado por:

$$
E\left[\theta_{h}\right]=\frac{\sum_{h=1}^{H} \theta_{h}}{H}
$$

\section{Demostración}

El problema del diseñador de política económica es: 
$\operatorname{Max}_{\left\{x_{h}^{*}\right\}} \Psi=\sum_{h=1}^{H} v_{h}=\sum_{h=1}^{H} \ln \left(x_{h}\right)-\sum_{h=1}^{H} \theta_{h} x_{h}-p_{x} \sum_{h=1}^{H} x_{h}$

Las condiciones de primer orden están dadas por:

$$
\frac{\partial \delta}{\partial x_{h}}=\frac{1}{x_{h}}-\sum_{h=1}^{H} \theta_{h}-p_{x}=0
$$

De tal forma que:

$x_{h}^{*}=\frac{1}{p_{x}+\sum_{h=1}^{H} \theta_{h}} \quad \forall h=1,2 \ldots . H$

Lo que implica el nivel de equilibrio agregado Pareto eficiente del bien $X^{*}$ es:

$$
X^{*}=\sum_{h=1}^{H} x_{h}^{*}=\frac{H}{p_{x}+\sum_{h=1}^{H} \theta_{h}}=\frac{1}{\frac{p_{x}}{H}+E\left[\theta_{h}\right]}
$$

Proposición 3. El nivel de impuestos que induce la asignación Pareto eficiente en una economía de mercado es:

$$
\tau_{h}^{*}=\sum_{h=1}^{H} \theta_{h}-\theta_{h}
$$

Considere ahora el problema de La familia $h$ determinado por

$$
\operatorname{Max}_{\left\{x_{h}^{*}\right\}} v_{h}=\ln \left(x_{h}\right)-\theta_{h} x_{h}-\left\{p_{x}+\tau_{h}^{*}\right\} x_{h}
$$

Lo que implica que el nivel de consumo de mercado para la familia tipo $h$ dado por $\tilde{x}_{h}^{m}$ tal que

$$
\tilde{x}_{h}^{m}=\frac{1}{p_{x}+\theta_{h}+\tau_{h}^{*}}=\frac{1}{p_{x}+\sum_{h=1}^{H} \theta_{h}} \quad \forall h=1,2 \ldots . H
$$

Lo que implica un nivel de equilibrio de mercado dado por: 
Raúl Alberto Ponce Rodríguez y Alan Adrián Rodríguez Hernández

$$
\tilde{X}^{m}=\sum_{h=1}^{H} \tilde{x}_{h}^{m}=\sum_{h=1}^{H} \frac{1}{p_{x}+\theta_{h}+\tau_{h}^{*}}=\frac{1}{\frac{p_{x}}{H}+E\left[\theta_{h}\right]}
$$

De tal forma aue una economía de mercado con un impuesto Piguviano $\tau_{h}^{*}=\sum_{h=1}^{H} \theta_{h}-\theta_{h}$ implica que este equilibrio es Pareto Eficiente ya que

$$
\tilde{X}^{m}=X^{*}
$$

\section{Demostración}

Considere ahora el problema de La familia $h$ determinado por

$$
\operatorname{Max}_{\left\{x_{h}^{*}\right\}} \quad v_{h}=\ln \left(x_{h}\right)-\theta_{h} x_{h}-\left\{p_{x}+\tau_{h}^{*}\right\} x_{h} \quad \forall h=1, \ldots H
$$

Lo aue implica que el nivel de consumo de mercado para la familia tipo $h$ por $\tilde{x}_{h}^{m}$ tal que

$$
\tilde{x}_{h}^{m}=\frac{1}{p_{x}+\theta_{h}+\tau_{h}^{*}}=\frac{1}{p_{x}+\sum_{h=1}^{H} \theta_{h}} \quad \forall h=1,2 \ldots . H
$$

Lo que implica un nivel de equilibrio de mercado dado por:

$$
\tilde{X}^{m}=\sum_{h=1}^{H} \tilde{x}_{h}^{m}=\sum_{h=1}^{H} \frac{1}{p_{x}+\theta_{h}+\tau_{h}^{*}}=\sum_{h=1}^{H}\left\{\frac{1}{p_{x}+\sum_{h=1}^{H} \theta_{h}}\right\}=\frac{1}{\frac{p_{x}}{H}+E\left[\theta_{h}\right]}
$$

Donde $E\left[\theta_{h}\right]$ es el costo personal promedio de la contaminación del bien privado, el cual está dado por $E\left[\theta_{h}\right]=\frac{\sum_{h=1}^{H} \theta_{h}}{H}$. 
Sistemas electorales y su influencia en el diseño de la política ambiental

Gráfica A1. El equilibrio electoral para una economía con un sistema electoral mayoritario

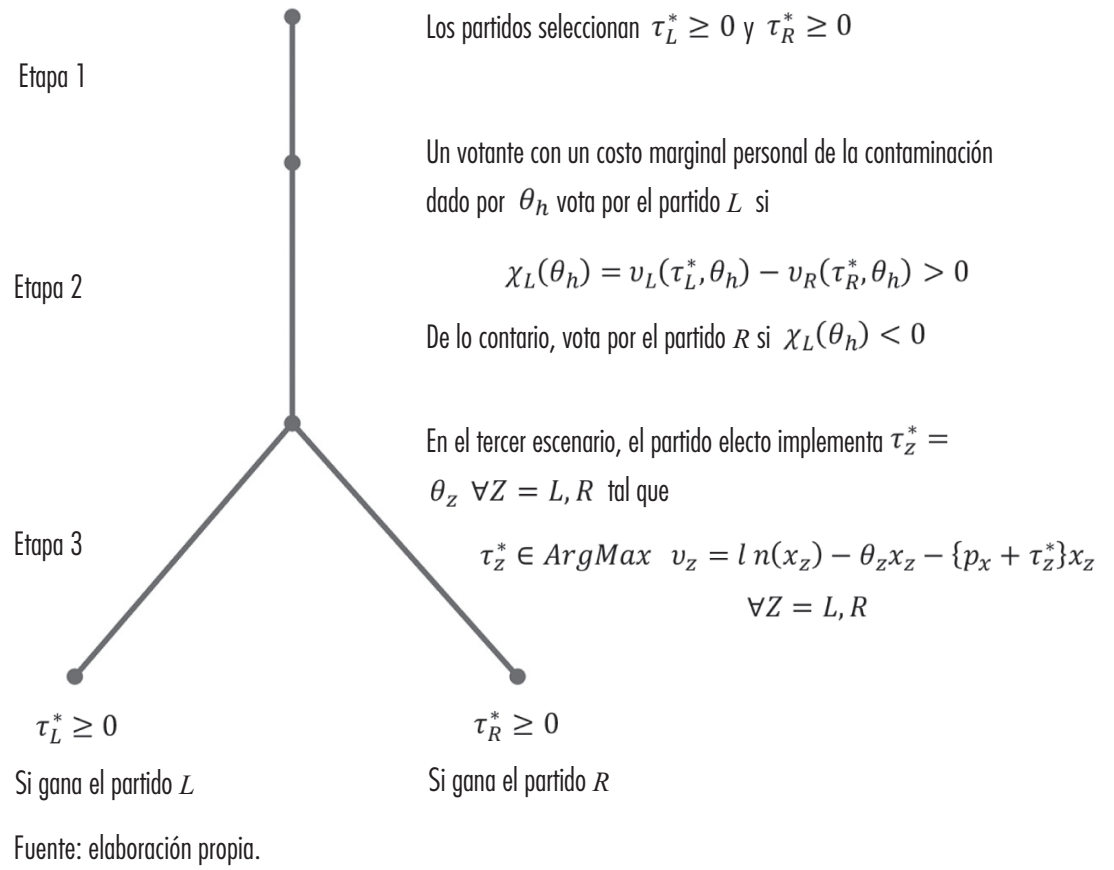


Gráfica A2. El equilibrio electoral para una economía con un sistema electoral proporcional

\begin{tabular}{|c|c|}
\hline Etapa 1 & Los partidos seleccionan $\tau_{L}^{*} \geq 0, \tau_{M}^{*} \geq 0 \mathrm{y} \tau_{R}^{*} \geq 0$ \\
\hline Etapa 2 & $\begin{array}{l}\text { En la segunda etapa del juego, el votante con } \theta_{h} \text { vota por el } \\
\text { partido } L \text { si } \chi_{L R}\left(\theta_{h}\right)>0 \text { y } \chi_{L M}\left(\theta_{h}\right)>0 \\
\text { por el partido } M \text { si } \chi_{M R}\left(\theta_{h}\right)>0 \text { y } \chi_{M L}\left(\theta_{h}\right)>0 \\
\text { por el partido } R \text { si } \chi_{R M}\left(\theta_{h}\right)>0 \text { y } \chi_{R L}\left(\theta_{h}\right)>0\end{array}$ \\
\hline Etapa 3 & $\begin{array}{l}\text { En la tercera etapa, los partidos reciben una proporción } \Omega_{\mathrm{z}} \text { de los asientos } \\
\text { del Congreso tal que } \Omega_{L}, \Omega_{M}, \Omega_{R} \in[0,1]: \Omega_{L}+\Omega_{M}+\Omega_{R}=1\end{array}$ \\
\hline Etapa 4 & 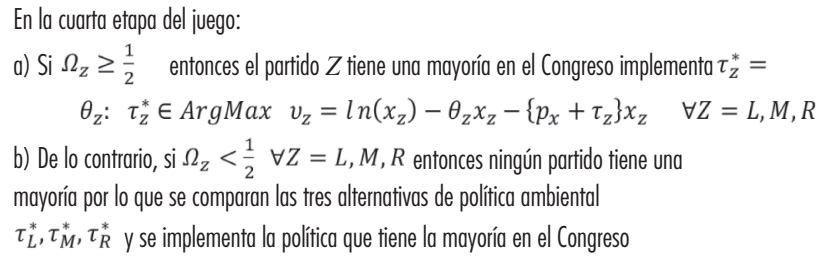 \\
\hline
\end{tabular}

Fuente: elaboración propia. 